\title{
THE PROBLEM OF THE CUBIC VARIETY IN $S_{4}^{*}$
}

\section{BY VIRGIL SNYDER}

1. Introduction. One of the outstanding problems of algebraic geometry that is still unsolved is the classification of Cremona transformations in space of more than two dimensions, and another, closely related to the preceding, is that of possible series of composition.

The theorem of Noether (80), later clarified and interpreted by Segre (90), Castelnuovo (14), Chisini (17), and Alexander (1), has answered the latter problem for the plane; while a large number of papers, represented in particular by the recent work of Hudson (67), Mlodzieioski (73)-(76), and Montesano (79), have made the construction of tables a definite procedure.

The smaller problem of the involutorial transformations has been completely solved by Noether (81), Bertini (6), and others, thus furnishing a weapon of incalculable importance for the study of various applications. Periodic transformations and their groups have been studied from various points of view by Kantor (69)-(70), Wiman (112), and Coble (20)-(21).

The corresponding problem in space is still almost undeveloped. Notwithstanding the excellent report made by Coble (22), the less ambitious one by me (103), the appearance of the extensive treatise by Hudson (67), and the Report of a committee of the National Research Council (106), I wish to speak of one phase of the latter problem. For regular transformations in space of three dimensions, the important theorem of Hudson (66) and a recent memoir of Montesano (78) are distinct steps in advance. When the

* Presidential address delivered before the Society, August 29, 1929. Written under the auspices of the Heckscher Foundation for the Promotion of Research, established by August Heckscher at Cornell University. 
defining elements are chosen independently, without contact conditions, the table of characteristics for a Cremona transformation and for its inverse has been completely constructed.

I wish to confine my attention, however, to the part taken by the cubic variety of four-way space $S_{4}$ in the study of involutorial birational transformations of $S_{3}$.

The study of the involution defined by the web of quadrics through six points leads at once to the Weddle surface, the locus of the vertex of the quadric cones through the six points, as surface of coincident points. If the quadrics of the web be made projective with the planes of another space $S_{3}^{\prime}$, a $(1,2)$ correspondence is established, and the Kummer surface appears as surface of branch points in $S_{3}^{\prime}$. All the properties of the general transformation and of all particularizations can be made from this point of view. This study of $(1,2)$ correspondences is an extension of that initiated by De Paolis (27) for $S_{3}$, and applied to double planes by Enriques (32) and by Castelnuovo and Enriques (15), thus confirming the result obtained by Noether (81) concerning the number of plane involutions of order two.

But the Kummer surface is the apparent contour of the cubic variety with ten nodes, as has been shown by Segre and further developed by Hudson (68) and by Snyder (101). Moreover, the same surface also appears in a similar rôle in connection with involutions defined by webs of cubic and of certain quartic surfaces. See Sharpe and Snyder (100).

In the same way, the quartic surface with less than sixteen nodes, birationally equivalent to the focal surface of line congruences of order two and class 3, 4, 5, 6 appear as surfaces of branch points of certain involutions, and each is the apparent contour of a cubic variety with one or more actual double points. Thus, to a point on the cubic variety correspond a pair of points in $S_{3}$, each point of which uniquely determines the other. These pairs of points determine an involution belonging to $V_{3}$. 
2. Rational Involutions. Given an algebraic curve $C$ in $S_{n}$, a necessary and sufficient condition that $C$ is rational is that its points can be put into $(1,1)$ continuous correspondence with those of a straight line. (Definition.)

If $\rho x_{i}=f_{i}\left(y_{1}, y_{2}\right),(i=0,1, \cdots, n)$, define the coordinates $(x)$ of a point on $C(x)$ and $y_{1}, y_{2}$ those of the image point on the line, then $f_{i}$ is a rational integral polynomial homogeneous in $y_{1}, y_{2}$.

If several sets of values of $y_{1}, y_{2}$ define the same variable point $(x)$, then functions $y_{1}=\phi_{1}\left(y_{1}^{\prime}, y_{2}^{\prime}\right), y_{2}=\phi_{2}\left(y_{1}^{\prime}, y_{2}^{\prime}\right)$ can be found such that the relation between $\left(y^{\prime}\right)$ and $(x)$ is $(1,1)$. This is expressed by saying that every involution on a rational curve is rational. (Theorem of Lüroth (71)).

Similarly, given an algebraic surface $F(x)$ in $S_{n}$, such that $\rho x_{i}=f_{i}\left(y_{1}, y_{2}, y_{3}\right)$, wherein each $f_{i}$ is a rational integral form of the same degree in $(x)$, then $F(x)$ is rational. If several sets of values of $(y)$ define the same variable $(x)$, then functions $y_{i}=\phi_{i}\left(y_{1}^{\prime}, y_{2}^{\prime}, y_{3}^{\prime}\right)$ can be found such that the relation between $\left(y^{\prime}\right)$ and $(x)$ is $(1,1)$. Hence every involution on a rational surface or in particular on a plane, is rational. (Theorem of Castelnuovo (11).) The theorem for involutions of order two was already known. See Noether (81) and Bertini (6). More recent contributions to the classification of plane involutions of order higher than two have been made by Howe (65), Hollcroft (64), and Sharpe (99).

Given a three-dimensional manifold $M_{3}(x)$ and $\rho x_{i}$ $=f_{i}\left(y_{1}, y_{2}, y_{3}, y_{4}\right)$ a parametric representation, wherein $f_{i}$ is a rational integral form, we cannot conclude whether $M_{3}$ is rational or not. The condition is necessary but it is not sufficient. If various sets of values $(y)$ define the same variable set $(x)$, it may not be possible to find a set of functions $y_{i}=\phi_{i}\left(y_{1}^{\prime}, y_{2}^{\prime}, y_{3}^{\prime}, y_{4}^{\prime}\right)$ such that the relation between $(x)$ on $M_{3}(x)$ and $\left(y^{\prime}\right)$ in $S_{3}^{\prime}$ is $(1,1)$. (Theorem of Enriques (36).)

Illustrations have been given of irrational involutions in $S_{3}$ belonging to a three-dimensional variety; others were furnished by Aprile (2) but all are of order larger than two. No illustrations have been found of three-dimensional in- 
volutions of order two that are known to be irrational.

In particular, it is not known whether the points of the general cubic variety $V_{3}$ in $S_{4}$ can be put into $(1,1)$ correspondence with those of $S_{3}$.

It is known that an involution of order two in $S_{3}$ belongs to it. The existence of this involution was known to Noether, and later proved by Segre (89) and by Marletta (72). The actual form of the parametric represention is

$$
\begin{aligned}
& \rho x_{1}=y_{1}^{2}\left(2 y_{1}^{3} y_{3} y_{4}+A y_{3}+B y_{1}\right), \\
& \rho x_{2}=y_{1} y_{2}(\quad \text { " }) \text {, } \\
& \rho x_{3}=-y_{3}{ }^{2}(\quad \text { " }) \text {, } \\
& \rho x_{4}=y_{1}\left(y_{1}^{3} y_{3} y_{4}^{2}+B y_{1} y_{4}-C y_{3}\right), \\
& \rho x_{5}=-y_{1}\left(y_{1} y_{4}^{2}+A y_{1} y_{4}+C y_{1}\right) \text {, } \\
& V_{3}=x_{1} x_{4}^{2}+x_{3} x_{5}^{2}+a x_{4}+b x_{5}+C=0 \text {, } \\
& a=a_{0} x_{3}{ }^{2}+a_{1} x_{3}+a_{2}, \quad A=a_{0} y_{3}{ }^{4}-a_{1} y_{3}{ }^{2} y_{1}+a_{2} y_{1}{ }^{2}, \\
& b=b_{0} x_{3}{ }^{2}+b_{1} x_{3}+b_{2}, \quad B=b_{0} y_{3}{ }^{4}-b_{1} y_{3}{ }^{2} y_{1}+b_{2} y_{1}{ }^{2} \text {, } \\
& c=c_{1} x_{3}{ }^{2}+c_{2} x_{3}+c_{3}, \quad C=c_{1} y_{3}{ }^{4}-c_{2} y_{3}{ }^{2} y_{1}+c_{3} y_{1}{ }^{2} \text {, }
\end{aligned}
$$

where $a_{i}, b_{i}, c_{i}$ are binary forms of order $i$ in $x_{1}$ and $x_{2}$.

This form of order 7 is the simplest one consistent with a quadratic locus of invariant points. This result was obtained and the associated involution of order 6 is described by Snyder (104).

3. Involutions Belonging Multiply to a Line Complex. Every $V_{3}$ contains $\infty^{2}$ straight lines. See Enriques (28) and Fano (57)-(58). Let $l$ be a line on $V_{3}$, and $P$ any point on $V_{3}$.

The plane $(P, l)$ meets $V_{3}$ in a residual conic through $P$ which meets $l$ in two points $Z_{1}, Z_{2}$. The line $P Z_{i}$ is tangent to $V_{3}$ at $Z_{i}$. Conversely, any line tangent to $V_{3}$ at a point $Z$ on $l$ meets $V_{3}$ again in a point $P$.

Between the points $P$ of $V_{3}$ and the tangents at points on $l$ there is therefore a $(1,2)$ correspondence. These tangent lines form a special linear line complex which can be mapped birationally on the points of an $S_{3}$. The lines joining a pair of associated points $P P^{\prime}$ in $S_{3}$ always meet the rectilinear image 
of $l$; hence they define a special linear complex. But every line of the complex contains two pairs of associated points. (Snyder (104).)

Other $I_{2}$ in $S_{3}$ exist which are contained multiply in a rational complex. The simplest one is obtained by constructing a $(1, k)$ correspondence between a pair of lines on a regulus and the quadrics of a pencil. A point $P$ of $S_{3}$ determines a quadric passing through it, and hence a pair of directrices $\left(d, d^{\prime}\right)$ of the regulus. The transversal of $\left(d, d^{\prime}\right)$ through $P$ meets the quadric in $P^{\prime}$. The line $P P^{\prime}$ contains $k$ pairs of associated points, and belongs to a general linear complex. (Snyder (107).)

Consider a line , and a pencil of surfaces of order $n$ having the line to multip', city $n-2$. Make the same $(1, k)$ correspondence between the points of the line and the surfaces of the pencil. The line may be replaced by a conic provided the surface is of ordes less than 5 , or by a space cubic.

A pencil of quadrics and a rational curve of any order may be taken. Similarly, a linear system consisting of a line and of a rational curve of order $m$ meeting it in $m-1$ points may be put in $(1, k)$ correspondence with the quadrics of a pencil. Either the line or the curve may remain fixed. The system may be replaced by a pencil of cubic curves as directrices. All of these forms contain types which belong to the cubic variety as particular cases. Incidentally they all possess properties not shared by $\mathrm{c}$ ther involutions heretofore known.

Another illustration is hat of order 7 found by Montesano (77). Given five linear ine complexes $K_{i}$ and a projective form $q$ or $g$ of five elemelit s. An arbitrary plane $\pi$ of space has five poles $O_{i}$ as to $K_{i}$, an 1 in it is one point $O^{\prime}$ such that the lines $O^{\prime} O_{i} \pi g$. There is one and only one complex $K$ in involution with every $K_{i}$. The plane $\pi$ has a pole $O$ as to $K$. The relation between $O, O^{\prime}$ is birational and involutorial.

The line $O O^{\prime}$ describes a linear complex $K$ and every line of $K$ contains three pairs of conjugate points. There is no surface of invariant points, but a curve of order 8 and genus 5 , birationally equivalent to the only fundamental curve of 
the system, apart from parasitic lines. It is not known whether this involution belongs to the cubic variety or not. Its properties are different from those of the other types just mentioned.

In particular, if the set of complexes be considered, such that each new one is in involution with five of the given ones $\left(K_{i}, K\right)$, and the corresponding transformation be considered, the rôles of the fundamental curve and of the curve of invariant points will be interchanged. The scheme really furnishes $\infty^{2}$ pairs of such complementary involutions, corresponding to the $\infty^{2}$ possible values of $g$.

4. Infinite Discontinuous Groups. Various infinite discontinuous groups which belong to $V_{3}$ exist. We shall consider six distinct generating operations numbered (a), (b), (c), (d), (e), (f) below. It has not been shown whether others also exist.

(a). The projection of $V_{3}$ upon itself from a vertex $A$ upon it. The product $A B$ of two such projections is not periodic, nor can it be replaced by another product. Similarly for the product of three or more such projections.

The projection $A$ is Cremonian, and quadratic. An $S_{3}$ is transformed into a quadric variety having the polar quadric of $A$ for locus of invariant points. The hyperplanar sections of $V_{3}$ are transformed into sextic surfaces all passing through the six lines of $V_{3}$ which pass through $A$. These lines have the property that the image of a point on any one of them is the whole line passing through it.

In the product $A B$ the $S_{3}$ sections of $V_{3}$ are transformed into surfaces of order 12, having six double basis lines through $B$, and six conics, all lying on a quartic surface, through another point, the image of $A$ in the operation $B$. Similarly for products of more projections $A, B, C \cdots$. (Snyder (102).)

(b). Let $l$ be a fixed line on $V_{3}$. A point $P$ of $V_{3}$ and $l$ determine a plane which meets $V_{3}$ in a residual conic passing through $P$. 
The line $l$ has a pole $L$ as to this conic. The harmonic homology defined by $L$ and $l$ leaves the conic, and hence also $V_{3}$, invariant. The transformation is Cremonian, of order 8 . The conjugate of an $S_{3}$ contains $l$ to multiplicity 7 . The locus of invariant points is a variety of order 5 , containing $l$ to multiplicity 4 . The image of $l$ itself is a variety of order 7, containing $l$ to multiplicity 6 . Every $S_{3}$ through $l$ is transformed into itself.

There are $\infty^{2}$ lines on $V_{3}$, each of which has an associated involution (b). The product of two such involutions, associated with different lines, is not periodic, nor can one such product be replaced by another when the lines are taken arbitrarily.

The product of a transformation of the type (a) and a transformation of the type (b) is in general not periodic. (Snyder (102).)

(c). Consider a pencil of $S_{3}$ having $\pi: \quad(a x)=0,(b x)=0$ for base, and the line $l \equiv x_{1}=x_{2}=x_{3}=0$ on $V_{3}$. Make the points of the line and the hyperplanes of the pencil projective, by associating each $S_{3}$ with the point $P$ in which it meets $l$. Project the cubic surface $S_{3}, V$ into itself from $P$. The transformation is Cremonian, of order 4, the fundamental elements are $l$ taken simply, and the basis plane taken twice. The locus of invariant points is a cubic variety containing $l, \pi$ each simply.

A transformation of this kind exists for every line $l$ on $V$, and when $l$ has been fixed, the basis plane may be chosen in $\infty^{6}$ ways. The product of two or more transformations of this type is not periodic. The product of any transformation of type (c) with one of type (a) or of type (b) or any sequence of $(a),(b),(c)$ is in general not periodic.

The line $l$ may be replaced by a conic, provided the basis plane has one point on it. The variety $V_{3}$ contains $\infty^{4}$ conics. Moreover, a space cubic with two points on the basis plane or a rational quartic with all three basis points upon it, may be taken. The directrix curve appears as a basis curve with multiplicity equal to its order in every case. 
(d). Assume an arbitrary fixed line $m$ through a point $M$ on $V_{3}$, but not lying on $V_{3}$. A point $P$ of $V_{3}$ and the line $m$ determine a plane which meets $V$ in a cubic curve through $M$. The first tangential $M^{\prime}$ of $M$, joined to $P$, meets $V_{3}$ in $P^{\prime}$. The transformation $P, P^{\prime}$ is involutorial and Cremonian of order 8. The locus of $M^{\prime}$ is the surface of intersection of $V_{3}$ and the three-dimensional tangent space at $M$, of order 3 . Every plane through $m$ is transformed into itself. The transformation in each such plane is quadratic, a perspective Jonquières type of the third kind. The fundamental line is the tangent to the cubic curve at $M^{\prime}$, and the locus of invariant points is the polar conic of $M^{\prime}$ as to the cubic. The images of the hyperplane sections $|x|$ of $V_{3}$ are surfaces of order $24,|8 x|$, having $M$ to multiplicity 6 , and the six lines of $V$ through $M$ to multiplicity 2. They also contain the locus of the first tangential of $M^{\prime}$ as the plane describes the linear system through $m$.

The product of two transformations of type (d) is not periodic. There are $\infty^{3}$ points on $V_{3}$, and $\infty^{3}$ directions $m$ associated with each point. The properties of this transformation should be further studied, both in $S_{4}$ and in $S_{3}$. Many of them are different from those of involutions heretofore known. The product of a transformation of type (d) with any one of a preceding type or one derived from any combination of the preceding types is in general not periodic.

(e). Let $l, l^{\prime}$ be two skew lines on $V_{3}$ and $\pi$ a plane not meeting either. The plane is the basis of a pencil of $S_{3}$. The $S_{3}$ through a point $P$ meets $\left(l, l^{\prime}\right)$ in $\left(K, K^{\prime}\right)$. The line $\left(K, K^{\prime}\right)$ meets $V$ in $M$. The line $P M$ meets $V_{3}$ in $P^{\prime}$. The transformation is involutorial, and consists in interchanging $P$ and $P^{\prime}$. The locus of $M$ is a rational $C_{4}$. The lines $l$ and $l^{\prime}$ lie in a space $\Sigma_{3}$ which cuts $V_{3}$ in a cubic surface $F_{3}$ containing $l$ and $l^{\prime}$. The pencil $\left(S_{3}\right)$ meets $\left(l, l^{\prime}\right)$ in ranges projective with it, hence with each other, hence $\left(K, K^{\prime}\right)$ describes a quadric surface $H_{2}$ in $\Sigma_{3}$, through $\left(l, l^{\prime}\right)$. The residual intersection of $F_{3}$ and $H_{2}$ is a rational $C_{4}$ which meets $\left(l, l^{\prime}\right)$ each in three points. 
Either or both lines may be replaced by conics skew to each other, provided $\pi$ meets each conic in one point, or one may be replaced by a cubic with two points on $\pi$, with the other directrix either a line skew to $\pi$ or a conic with one point on $\pi$. When both directrices are cubics, they must have a common point on $\pi$. Corresponding forms exist in a three-way space which leave a given cubic surface invariant, each of which presents interesting configurations of fundamental elements.

(f). Another transformation is the following. Given a line $l$ on $V_{3}$ and a plane $\pi$ of $S_{4}$, skew to $l$.

The points $O$ of $\pi$ and the planes through $l$ are projective, and perspective. The plane through $l$ meets $V_{3}$ in a residual conic. Consider the harmonic homology having $O$ for center and its pole as to the conic in its associated plane for axis. This procedure defines an involutorial birational transformation which leaves each conic of $l$ in a plane through $l$ invariant, and hence transforms $V_{3}$ into itself.

It is Cremonian. The line $l$ and the plane cubic curve section of $V_{3}$ by $\pi$ are the fundamental elements. The plane $\pi$ may be replaced by any rational surface meeting an arbitrary plane through $l$ in one point apart from those on $l$.

5. Continuous Groups. A curve of genus zero belongs to a three-parameter group of continuous transformations. One of genus 1 has $\infty^{1}$ transformations which do not form a group. If the genus exceeds unity, the curve belongs at most to a finite group. Surfaces invariant under $\infty^{1}$ linear transformations are rational or reducible to ruled surfaces. If they are invariant under $\infty^{2}$ such transformations they are necessarily rational. (Fano (40).) Every algebraic surface, invariant under a transitive continuous group of linear transformations, can be transformed birationally into a plane, or a quadric of $S_{3}$, or into a rational cone normal in a certain $S_{m+1}$ in such a manner that the group considered gives rise respectively to a group of plane homographies, or to a group of homographies in $S_{3}$, or in $S_{m+1}$, each leaving the associated form invariant. (Fano (41) and (44).) 
Every three-parameter linear group of $S_{3}$ leaves a series of forms generated by planes invariant, none containing a simpler one. (Fano (40).)

The three-dimensional varieties of $S_{4}$ invariant under $\infty^{4}$ continuous linear transformations are classified into five types, which do not include the general cubic variety. (Fano (43).)

Surfaces invariant under a continuous group of Cremona transformations are all reducible to rational surfaces in $S_{3}$ invariant under linear transformations. (Fano (45).) Similarly for three-dimensional varieties. (Fano (46).)

Given a homogeneous linear differential equation of order $n$. If a system of solutions $y_{1}, \cdots, y_{n}$ be regarded as projective coordinates of a point of $S_{n-1}$, the point will describe a rational curve when the coefficients in the given equation are rational functions of $x$. If the (y) satisfy one or more algebraic relations, the curve will lie on a corresponding number of varieties. The group of rationality of the equation leaves each variety of the system invariant. Finite groups of Cremona transformations in the plane, both according to the order and to the type, in the sense of Kantor and of Wiman have all been determined in this way. (Enriques and Fano (38).) The types of continuous groups in the plane are projective, quadratic and Jonquières, and in $S_{3}$ contain these and two other $\infty^{3}$ types. The generalized Jonquières groups leave a pencil of planes or a bundle of lines (or both) invariant. (47).

The imprimitive groups of continuous Cremona transformations in $S_{3}$ belong as subcases in the types derived by Enriques (29) and Fano (46), (48).

The complete enumeration of continuous Cremona transformations in $S_{3}$ can be made by starting from the generating infinitesimal transformation. This has been done and the preceding results confirmed. (49).

A differential equation of the type in question is always solvable when more than one algebraic equation is identically satisfied by the solutions. (50). 
The group of rationality may leave a quadratic form in $S_{n-1}$ invariant. (52). For $n=6$, this case can be interpreted in terms of lines of $S_{3}$; instead of a curve, the locus is now a ruled surface. For $n=5$, the same interpretation can be made by assuming a linear relation among six solutions. The ruled surface is then contained in a linear line complex. (51). If the discriminant of the quadratic form vanishes (special complex), the equation can be reduced to one of order 4. (54).

Every $M_{3}$ invariant under $\infty^{3}$ projective transformations is rational. If the group is not integrable, the existence of $\infty^{1}$ rational surfaces on it insures its rationality. (53).

These results have been collected and systematically developed by Fano (55). It is shown that the existence of algebraic relations among the fundamental solutions of a linear differential equation is a necessary and sufficient condition for the vanishing of certain invariants belonging to the equation.

On the general quartic three-dimensional variety $V_{3}^{4}$ of $S_{4}$, without double points, the system of hyperplane sections is the only linear system of regular surfaces with genera equal to unity and of dimension not less than 2 . This variety is not invariant under any birational transformation, with the possible exception of linear ones.

The $M_{3}{ }^{6}$ of $S_{5}$, complete intersection of $V_{4}{ }^{2}$ and $V_{4}{ }^{3}$, does not contain such surfaces either. Every birational transformation which leaves it invariant is the product of a finite number of double projections from lines lying on it and of possible linear transformations. (Fano (61).)

6. Invariants. The question naturally arises whether algebraic or topological invariants exist, particular values of which can indicate the rationality or irrationality of $V_{3}$.

A necessary and sufficient condition that an irreducible algebraic curve in space of any number of dimensions shall be rational is that its genus is zero. (Clebsch (18).)

An algebraic surface has an infinite number of invariants analogous to the genus of a curve, but a necessary and 
sufficient condition that a surface shall be rational is that its arithmetic genus $p_{n}$ and its bigenus $P_{2}$ should both be zero. (Castelnuovo (13).) That a surface may have both its geometric genus and its arithmetic genus equal to zero and still not be rational, was shown by Enriques (35).

A system of invariants of three-dimensional varieties was determined by Pannelli (84), analogous to the genera of a surface. (Castelnuovo and Enriques (16).) Some of the fundamental concepts of invariants on hyperdimensional manifolds had been discussed by Noether (82). It was then shown by Severi (97) that all these invariants vanish for the cubic variety of $S_{4}$, also for the general quartic $V_{3}^{4}$ of $S_{4}$, and for the $M_{3}^{6} \equiv\left(V_{4}^{2}, V_{4}^{3}\right)$ of $S_{5}$, hence that the various indices of irregularity are zero. These latter $V_{3}^{4}$, $M_{3}{ }^{6}$ contain only complete intersections. (Severi (92), Fano (59).)

The algebraic surfaces contained in $V_{3}^{3}$ were obtained by Fano (56).

The $M_{3}{ }^{6}$ is the representative of the general cubic line complex of $S_{3}$, which has been extensively studied by Voss (111) and by Veneroni (109). In particular, it has $\infty^{1}$ linear pencils of lines or quadric reguli, hence $M_{3}{ }^{6}$ contains $\infty^{1}$ straight lines. By projecting the manifold from one of them, it can be mapped on $S_{3}$ doubly, with a sextic surface of branch points. The two forms, $V_{3}{ }^{4}$ and $M_{3}{ }^{6}$, are birationally distinct. (Fano (59).)

The assumption that either contains a homaloidal system of surfaces leads to a contradiction; hence both of these forms are irrational, that is, they can not be mapped birationally on $S_{3}$. (Fano (59).) However, both can be mapped on $S_{3}$ by means of an involution that is rational in one sense only.

The actual process was outlined for $M_{3}{ }^{6}$ by Enriques (36), which establishes the important result that irrational involutions in $S_{3}$ exist. Other examples of involutions belonging to three-dimensional varieties were given by Marletta (72), and Aprile (2) showed that the Enriques involution is of 
order 216, but that the manifold can be mapped on $S_{3}$ by means of an involution of order 36. Further conditions for rationality were found by Enriques (37).

These forms are much more general than $V_{3}{ }^{3}$. The general $M_{3}{ }^{6}$ does not contain any planes, since the general cubic line complex of $S_{3}$ does not contain a bundle of lines. If now the complex be particularized to contain three distinct bundles of lines (or dually, three plane fields of lines), then the $M_{3}{ }^{6}$ contains three planes belonging to the same system on the quadric variety passing through it. This particularized $M_{3}{ }^{6}$ is birationally identical with the general $V_{3}^{3}$.

Similarly, the $V_{3}{ }^{4}$ can be particularized to the extent of having a double line and still be much more general than the general cubic variety. It may be desirable to study the properties of these particular forms in order to obtain those of the general $V_{3}^{3}$.

The two general forms $V_{3}{ }^{4}$ and $M_{3}{ }^{6}$ are included in the general category $M_{3}^{2}{ }^{p-2}$ of $S_{p+1}$ (Fano (62)) having surface sections regular with all their genera equal to unity, and curve sections canonical curves of genus $p . V_{3}^{4}$ corresponds to $p=3$ and $M_{3}{ }^{6}$ to $p=4$. The case $p=2$ appears as a double $S_{3}$ with a sextic surface of branch points, but this can be obtained by projecting $M_{3}{ }^{6}$ upon $S_{3}$ from one of its lines, in a particular form.

The case $p=5$ is represented by the intersection of three general quadratic varieties in $S_{6}$. It contains no surfaces other than complete intersections with other forms. This manifold can be mapped on $S_{3}$ by an involution of order 4 . It contains an infinite number of rational congruences of the first order, of rational curves.

The general $V_{3}{ }^{n}: S_{1}{ }^{n-2}$ of $S_{4}$ has been studied from various points of view, but most of the properties found for larger values of $n$ do not exist for $n=3$. (Enriques (33).)

As yet no topological invariants have been found that are characteristic of the general $V_{3}{ }^{3}$, normal in $S_{4}$.

Various properties regarding the analytical representation of a variety and of manifolds have been found by Severi 
(91), those regarding postulation (93), those concerning complete intersections of non-singular forms (94), (95).

7. Sections by $\left|S_{3}\right|$. If all the plane sections of a surface in $S_{3}$ are rational, the surface is rational. It is either ruled or is a Steiner quartic surface with three concurrent double lines. (Picard (85), Guccia (63).)

If the sections by $\left|S_{n-1}\right|$ of a manifold $M_{3}$ in $S_{n}$ are rational surfaces, $M_{3}$ is rational except possibly when $M_{3}$ is $V_{3}^{8}$ of $S_{4}$, concerning which no conclusion can be drawn, as the method does not apply. (Fano (60).)

Probably the transcendental methods of Bagnera-De Franchis (3)-(5), those of Enriques-Severi (3), and those of Severi (96), (98) as applied to hyperelliptic surfaces, may be extended to three-dimensional varieties. As yet no light has been thrown on $V_{3}^{3}$ from this source. Another possible method of extension is that of Comessatti (23) in his study of real rational surfaces.

8. Rational $V_{3}{ }^{3}$. Let there be given a cubic variety $V_{3}{ }^{3}(x)$ $=0$ in $S_{4}$, such that each $x_{i}$ may be expressed in the form $\rho x_{i}=f_{i}\left(y_{1}, y_{2}, y_{3}, y_{4}\right)$, each $f_{i}$ being a rational quaternary form of order $N$. When these values of $x_{i}$ are substituted in $V(x)=0$, it shall be identically satisfied, and that for an arbitrary set of values of the $y_{i}$; no other set can be found which will define the same value of $(x)$.

Since $\sum a_{i} x_{i}=0, V_{3}^{3}(x)=0$ define a cubic surface, it follows that the $f_{i}(y)$ must satisfy the following properties.

(a) The system is linear of dimensionality 4.

(b) Any two surfaces of the system intersect in a curve of genus 1. (Plane sections of a cubic surface.)

(c) Any three intersect in three variable points.

From any simple point $O_{x}$ on $V_{3}^{3}(x)$ project the variety on any space $S_{3} \equiv \pi$ not passing through $O_{x}$. In $\pi$, every point is the image of two points of $V_{3}^{3}$; those on the generators of the cone of apparent contour project into the points of the quartic surface of branch points $L_{4}(x) \equiv u_{2}{ }^{2}(x)-u_{1}(x) \cdot u_{3}(x)$ $=0$ in which $u_{1}=0$ is the tangent $S_{3}$ to $V_{3}^{3}$ at $O_{x}$. The plane 
$u_{1}=0$ in $\pi$ touches $L_{4}(x)=0$ along the conic $u_{2}=0$, which contains the six double points on $L_{4}(x)=0$, defined by $u_{1}=u_{2}=u_{3}=0$. Between the two three-spaces $\pi$ and $(y)$ there is a $(1,2)$ correspondence, in which $L_{4}(x)=0$ in $\pi$ is the surface of branch points.

Let the equation of $V_{3}^{3}$ be $u_{1} x_{5}{ }^{2}+2 u_{2} x_{5}+u_{3}=0$. Then the projection of $V_{3}^{3}$ upon $x_{5}=0$ as double $S_{3}$ from $O_{x} \equiv(0,0,0$, $0,1)$ has the surface $L_{4}(x)=0$ of branch points. Let $O_{y}$ be the image of $O_{x}$ supposed to be non-singular in $\rho x_{i}=f_{i}(y)$. Then $\left(y_{0}\right)$ satisfy the four equations $f_{i}(y)=0,(i=1,2,3,4)$.

In the $(1,2)$ correspondence between $(y)$ and $x_{5}=0$, the point $O_{5}$ is singular. In the projection upon $x_{5}=0$ from $O_{x}$, the conjugate of $O_{x}$ is the plane $u_{1}=0$; it is the intersection with $x_{1}=0$ of the tangent $S_{3}$ to $V_{3}^{3}$ at $O_{x}$. The conjugate of $O_{y}$ is then the $f$ of the $\infty^{4}$ system defined by $u_{1}(f)=0$.

The points of $u_{1}=0$ in $\pi$ are of three kinds, those not on $L_{4}$ are all conjugate to $O_{x}$; those on $u_{2}=0, u_{1}=0$ but not on $u_{3}=0$ are all branch points at $O_{x}$, as $u_{2}=0$ defines the quadric cone of inflexional tangents to $V_{3}$ at $O_{x}$.

Finally, the six double points $u_{1}=0, u_{2}=0, u_{3}=0$ are images in $x_{5}=0$ of the six lines of $V_{3}$ which pass through $O_{x}$.

Any $S_{3}$ passing through $O_{x}$ meets $V_{3}$ in a cubic surface which is transformed into itself by interchanging the two points of $V_{3}$ on every line through $O_{x}$.

Hence in $(y)$, each $f_{i}(i \neq 5)$ is transformed into itself. The curves $f_{k}=0, f_{i}=0,(i, k \neq 5)$ of genus 1 are the images of the lines of $S_{3}$.

The $\infty^{4}$ system $|f|$ is not invariant under $I_{2}$, and the subsystem is still of grade 3 when the fixed point $O_{v}$ is adjoined.

A section $\sum a_{i} x_{i}=0$ not passing through $O_{x}$ meets $V$ in a general cubic surface. The three-dimensional cone joining the surface to $O_{x}$ meets $V$ in a sextic surface, the complete intersection of $V$ and a quadratic variety $|2 x|$, obtained from $\sum a_{i} x_{i}$ by the quadratic transformation

$$
\left\{\begin{array}{l}
\rho x_{i}^{\prime}=u_{1} x_{i}, \\
\rho x_{5}^{\prime}=-u_{1} x_{5}-u_{2} .
\end{array} \quad(i=1, \cdots, 4)\right.
$$


The corresponding transformation in $(y)$ becomes

$$
\begin{aligned}
& \sigma f_{i}\left(y_{1}^{\prime}, \cdots, y_{4}^{\prime}\right)=u_{1}(f(y)) f_{i}(y), \\
& \sigma f_{5}\left(y_{1}^{\prime}, \cdots, y_{4}^{\prime}\right)=-u_{1}(f(y)) f_{5}(y)-u_{2}(f(y)),
\end{aligned}
$$

and for the involution $I_{2}$, of associated points,

$$
f_{i}\left(y^{\prime}\right)=f_{i}(y) \text {. }
$$

Thus, the quadratic image of any $S_{3}$ section in the transformation $T$ passes through the quadric surface $u_{1}=0$, $u_{2}=0$, which is the intersection of the tangent space to $V_{3}$ at $O_{x}$ and the quadric polar of $O_{x}$ as to $V_{3}$.

This is the quadric of inflexional tangents to $V_{3}$ at $O_{x}$. It meets $V_{3}$ in the six lines passing through $O_{x}$.

The images of these six lines are six curves through $O_{y}$, common to the conjugates of the $\infty^{4}|f|$. They are all parasitic curves.

Suppose $V_{3}{ }^{3}$ has a double point not lying in the tangent $S_{3}$ at the center of projection. Then in $x_{5}=0$ the surface of branch points $L_{4}=0$ has a double point not lying in the singular plane $u_{1}=0$ associated with the center of projection.

Conversely, if $L_{4}=0$ has a double point not lying in $u_{1}=0$, from the vanishing of the first derivatives of $L_{4}$ as to $x_{1}$, those of $V_{3}^{3}$ can be shown to vanish. Hence we may draw the following conclusion.

$A$ necessary and sufficient condition that the surface of branch points $L_{4}=0$ has a double point not in the singular plane associated with the center of projection is that $V_{3}{ }^{3}$ has a double point not in the tangent space at the center of projection.

If the values $x_{i}$ are substituted in $L_{4}$, the result is a perfect square $K^{2}(y)=0$ : the surface of contact of $V$ and its threedimensional tangent cone from $O_{x}$.

If $V_{3}{ }^{3}$ has a double point $P$ not at $O_{x}$, its image will be an actual double point of $L_{4}=0$, not on $u_{1}=0$. In this case, the parametric representation of $V_{3}^{3}: P^{2}$ can be obtained immediately by projecting $V_{3}{ }^{3}$ from $P$ and cutting the projecting cone by any $S_{3}$ not passing through $P$. 
If $u_{1} \equiv x_{1}$, and if $u_{2}$ and $u_{3}$ contain $x_{1}$ at most to the first power, then

$$
\begin{aligned}
V_{3} \equiv u_{1} x_{5}^{2}+ & 2 u_{2} x_{5}+u_{3} \\
= & x_{1} x_{5}{ }^{2}+2\left(x_{1} v_{1}+w_{2}\right) x_{5}+w_{3}=0 \equiv x_{1} t_{2}+t_{3},
\end{aligned}
$$

where $v_{i}, w_{i}$ are ternary in $x_{2}, x_{3}, x_{4}$ of degree $i$, and

$$
t_{2}=x_{5}^{2}+2 v_{1} x_{5}+v_{2}, \quad t_{3}=2 w_{2} x_{5}+w_{3},
$$

represents a cubic variety having a double point at the point $(1,0,0,0,0)$.

The representation in the space $(y)$ now takes the form

$$
\begin{aligned}
& \rho x_{1}=-t_{3}\left(y_{2}, \cdots, y_{5}\right), \\
& \rho x_{i}=y_{i} t_{2}\left(y_{2}, \cdots, y_{5}\right), \quad(i=2, \cdots, 5) .
\end{aligned}
$$

The image of the double point is the quadric surface $t_{2}=0$, and the fundamental element in $(y)$ is the sextic space curve $\gamma_{6}$ of genus $4, t_{2}=0, t_{3}=0$.

The $\infty^{4}$ linear system, images of the $S_{3}$ sections of $V_{3}$, now has the form

$$
\sum a_{i} y_{i} t_{2}-a_{1} t_{3}=0 ;
$$

thus it includes all the cubic surfaces passing through $\gamma_{6}$. This system satisfies conditions (a), (b) and (c).

The image in $(y)$ of the vertex $(0,0,0,0,1) \equiv O_{x}$ is the point $(0,0,0,1)$, hence the $\infty^{3}$ subsystem which defines the $(1,2)$ correspondence between $x_{5}=0$ and $(y)$ has the form

$$
\begin{aligned}
& \rho x_{1}=-2 w_{2}\left(y_{2}, y_{3}, y_{4}\right) y_{5}+w_{3}\left(y_{2}, y_{3}, y_{4}\right), \\
& \rho x_{2}=y_{2} t_{2}\left(y_{2}, \cdots, y_{5}\right), \\
& \rho x_{3}=y_{3} t_{2}\left(y_{2}, \cdots, y_{5}\right), \\
& \rho x_{4}=y_{4} t_{2}\left(y_{2}, \cdots, y_{5}\right), \\
& \sigma y_{2}=x_{1} x_{2}, \\
& \sigma y_{3}=x_{1} x_{3}, \\
& \sigma y_{4}=x_{1} x_{4}, \\
& \sigma y_{5}=-\left(v_{1} x_{1}+w_{2}\right) \pm\left[\left(v_{1} x_{2}+w_{2}\right)^{2}-x_{1}\left(x_{1} v_{2}+w_{3}\right)\right]^{1 / 2} .
\end{aligned}
$$


The equations of $I_{2}$ in $(y)$ can now be determined at once. The transformation is quartic, monoidal perspective. The surface of invariant points is

$$
w_{2} y_{5}^{2}-w_{3} y_{5}+v_{1} w_{3}+v_{2} w_{2}=0 .
$$

Now suppose that $V_{3}{ }^{3}$ is always rational, and that its equation contains a parameter $\lambda$, such that when $\lambda=0$ the corresponding variety acquires a double point at $P$. Then

$$
\begin{aligned}
& \rho x_{i}=\phi_{i}(y, \lambda)=y_{i} v_{2}(y) F(y)+\lambda f_{i}(y, \lambda),(i=2, \cdots, 5), \\
& \rho x_{1}=\phi_{1}(y, \lambda)=-t_{3}(y) F(y)+\lambda f(y, \lambda) .
\end{aligned}
$$

Consider any linear system of at least $\infty^{4}$ rational surfaces of order $N$. By using the common basis elements as part or all of the fundamental elements of the transforming system, suppose the system has been reduced to its simplest form. Then within the system construct a subsystem which satisfies conditions (a), (b), (c). Evidently, by any birational transformation of $(y)$, the properties of $V(x)$ will remain unchanged. Any rational surface may be transformed by means of its adjoints into one having plane sections of genus $0,1,2,3$ or of any positive genus, but hyperelliptic. (Enriques (33), (34).) We shall consider only those linear systems which belong to one or another of these five types, I, . . , V and which satisfy the conditions (a), (b), (c).

Finally, we select an additional basis point to define the $(1,2)$ correspondence. The five types will be considered in turn, but the three following properties will first be established.

9. Properties of Basis Elements. In the $\infty^{4}$ system $|f(y)|$, images of the spatial sections $|x|$ of $V_{3}^{3}$, is a subsystem $\infty^{3}|\bar{f}(y)|$ having an additional basis point not a basis point of the larger $\infty^{4}$ system. This special basis point is the image of an ordinary point $O_{x}$ on $V_{3}{ }^{3}$. If now $V_{3}{ }^{3}$ be projected upon a space $\pi$ not passing through $O_{x}$ from $O_{x}$ doubly, then between $\pi$ and $(y)$ exists a $(1,2)$ correspondence, such that each $\bar{f}(y)$ is transformed into 
itself, both images of a point in the image plane in $\pi$ being in this surface. In the projection of $V_{3}{ }^{3}$ from $O_{x}$ upon $\pi$, the fundamental element is the plane, which is the intersection of the tangent $S_{3}$ to $V_{3}^{3}$ at $O_{x}$ with $\pi$. The image of this plane in $(y)$ is a definite $\bar{f}$ of the subsystem.

If $|\bar{f}|$, and therefore $|f|$, has any other simple basis point, there is one surface $\bar{f}$ belonging to it having this point for double point; the point and the nodal surface are conjugate in the associated $I_{2}$ of $(y)$. The associated plane in $\pi$ must therefore touch $L$ at every common point, since its image in $(y)$ is composite; that is, the plane is a singular tangent plane of $L$ and hence contains six double points. In no cases can all these points be in the first singular tangent plane. Therefore we have the following results.

(A) If $|f|$ contains a simple isolated basis point, not that defining $|\bar{f}|, L$ has double points not in the characteristic singular plane.

Similarly, if any surface of the system $|\bar{f}|$ is composite, the two components are conjugate to each other in $I_{2}$, and the associated plane in $\pi$ must touch $L$ at every common point; that is, it is a singular plane; hence $L$ contains double points not in the singular plane $u_{1}$. (Sharpe and Snyder (100).)

(B) If $|\bar{f}|$ contains a composite surface, $L$ has double points not in the characteristic singular plane.

Let $\delta$ be a curve in $(y)$, which meets the surfaces of $|f|$ only in basis points or in points of basis curves. The surfaces of the system which pass through a point of $\delta$ must contain the whole curve. Hence $\infty^{2}$ surfaces of $|\bar{f}|$ contain $\delta$. The images of these surfaces are $\infty^{2}$ planes having the image of $\delta$ in common, hence it must be a point. The curve must lie entirely on $K$, the surface of coincident points of $(y)$, and the image point is a double point of $L$. (Segre (89).) If $\delta$ does not pass through $O_{y}$, this double point on $L$ can not be in the characteristic singular plane of $L$.

(C) If (y) contains a curve $\delta$ meeting $|f|$ only in basis points, 
and not passing through the basis point $O_{y}$ of $|\bar{f}|, L$ contains a double point not lying in its characteristic singular plane.

10. Rational Plane Sections. I. When the plane sections of a surface $f(y)=0$ are rational the system $|f|$ consists of ruled surfaces or of Steiner surfaces, that is, quartics with three concurrent double lines. (Picard (85), Guccia (63).) The simplest case satisfying conditions (a), (b), (c) is that of quadrics through 5 points. (Sharpe and Snyder (100).) The surface $L_{4}=0$ is now the 16-nodal Kummer surface, hence $V_{3}(x)=0$ is nodal. In case of surfaces of order greater than 2, the variable curve of intersection is always rational since the double curves of each $f_{i}$ must belong to the basis curve of the system. (Castelnuovo (12), Enriques (30).)

11. Plane Sections of Genus 1. II. The simplest case is $N=3$. Conditions (a), (b), (c) are satisfied; in every case $L_{4}=0$ has a number of extra nodes. (Sharpe and Snyder (100).) Compare the general properties of linear systems of plane curves of general genus. (Segre (88).)

$N=4$ gives a system of quartic surfaces with a double conic and certain other basis elements. But this can be reduced to the preceding one by a quadratic transformation. For $N=5$ the basis elements are a double $C_{5}$ with a triple point. The residual intersection of any two surfaces of the system is an elliptic quintic curve; the system is $\infty^{6}$ of grade 5. The conditions (a), (b), (c) will be satisfied only by imposing two additional points; hence $L_{4}$ will have another singular plane and $V_{3}^{3}$ has nodes.

For $N$ greater than 5 , there is no residual curve. (Del Pezzo (26).) For varieties with curve sections of genus 1, see Scorza (86).

12. Plane Sections of Genus $p=2$. III. This will be discussed in connection with general hyperelliptic sections. The surface defined by $\left|C_{6}\right|: 8 P^{2}$ is the double quadric cone, which can be mapped on a quartic surface with a double line, which is associated with a nodal variety. 
13. Plane Sections of Genus $p=3$. IV. The non-hyperelliptic case has been discussed by Castelnuovo (9) and by Scorza (87). Those reducible to quartics all have fundamental lines, hence they lead to nodal varieties. (Noether (83).)

Those of the first species, that is, those containing a net of rational curves of order 4 , meeting by twos in a single point, can in general not be reduced to quartics.

The only exception is that of the monoids, represented on the plane by $C_{4}: 12 P^{1}$, the 12 basis points all lying on a cubic curve, which is the image of the triple point. Whatever system of basis curves is adjoined to obtain a subsystem satisfying conditions (a), (b), (c), fundamental lines are introduced, hence the associated $V_{3}{ }^{3}$ has one or more double points. The rational quartic curves lie in the planes of the bundle having the triple point for vertex. For a discussion of the involutions which leave invariant a web of monoids, see Snyder (105).

The system $\left|C_{4}\right| \cdot 11 P^{1}$ defines a rational quintic surface with a double cubic curve. All the possible forms of residual basis elements that can satisfy conditions (a), (b), (c) are given in (100); in each $L_{4}$, and hence $V_{3}^{3}$, has double points.

14. Digression. Details of a Quintic Surface. Case $J$, Sharpe and Snyder (100), merits further study. The table of characteristics as given is incomplete.

Using the notation there employed, the line $h$ which is the partial image of $h^{\prime}$ in $\left(x^{\prime}\right)$, with which it is in $(1,1)$ correspondence, is double on each $S_{21}$ of the web, since $h^{\prime}$ is double on each $S_{6}^{\prime}$ of the system in $\left(x^{\prime}\right)$. Moreover, the curves on $\mathrm{H}_{2}$, which are the residual images of the points of $h^{\prime}$, are conics.

The curve $\beta_{5}$ meets $H_{2}$ in two points not on $\gamma_{3}$.

The line $l$ joining them is axis of a pencil of planes, each of which cuts from $\mathrm{H}_{2}$ a parasitic conic.

Two of these have for images in $\left(x^{\prime}\right)$ the points of contact of $h^{\prime}$ and $L_{4}^{\prime}$, hence the conics lie on $K_{10}$ and are double on 
each $S_{21}$ of the web. Each of the six lines $\left[R_{4}, K_{10}\right]=6 r$ also belongs to the base of $\left|S_{21}\right|$; hence we have

$$
\begin{array}{ll}
S_{21}: & \gamma_{3}^{8} \alpha^{4} \bar{\alpha}^{4} \beta_{5}^{5} 6 r \rho_{5} h^{2} 2 c_{2}{ }^{2}, \\
K_{10}: & \gamma_{3}^{4} \alpha^{2} \bar{\alpha}^{2} \beta_{5}{ }^{2} 6 r 2 c_{2} .
\end{array}
$$

A plane meets its conjugate $S_{21}$ in a composite curve consisting of $\left(S_{1}, K_{10}\right)$ and of a residual curve

$$
\delta_{11}: \quad \gamma_{3}^{4} \alpha^{2} \bar{\alpha}^{2} \beta_{5}^{3} \rho_{5} h^{2} 2 c_{2} .
$$

Any two $S_{21}$ may also have $x_{1}$ curves of order $i$, parasitic on the web $\left|S_{5}\right|$, and appearing to multiplicity $i$ on $\left|S_{21}\right|$. The complete intersection then gives

$441=21+192+16+16+125+6+5+4+16+\sum i^{3} x$, and the intersection with $K_{10}$ furnishes

$$
210=10+96+8+8+50+6+8+\sum i^{2} x_{i} .
$$

The only possible solution is $x_{1}=8, x_{2}=4$. The 8 lines are divided in to two groups of four each, bisecants of $\beta_{5}$ meeting $\gamma_{3}$ and $\alpha$ or $\bar{\alpha}$. Hence we may now write

$$
\begin{aligned}
S_{21}: & \gamma_{3}{ }^{8} \alpha^{4} \bar{\alpha}^{4} \beta_{5}{ }^{5} 6 r \rho_{5} h^{2} 2 c_{2}{ }^{2} 8 u_{1} 4 u_{2}{ }^{2}, \\
K_{10}: & \gamma_{3}^{4} \alpha^{2} \bar{\alpha}^{2} \beta_{5} 6 r 2 c_{2} 8 u_{1} 4 u_{2}, \\
\delta_{11}: & \gamma_{3}^{4} \alpha^{2} \bar{\alpha}^{2} \beta_{5}{ }^{3} \rho_{5} h^{2} 2 c_{2} 4 u_{2},
\end{aligned}
$$

where the interpretation of the symbols in the last equation undergoes an obvious modification.

The genus of $\delta_{11}$ is consequently 9 , and it intersects $K_{10}$ in 12 variable points. The images of these 12 points are the points of contact of $\delta^{\prime}$ and $L^{\prime}$; hence $\delta^{\prime}$, the variable double curve on $S_{6}^{\prime}$, image in $\left(x^{\prime}\right)$ of $S_{21}$, is of order 6 . The genus of $\delta^{\prime}$ is 2 .

The curve $\delta_{11}$ has a double point $D$ on $h$, and the plane of $\delta_{11}$ meets the image conic in two points $P_{1}$ and $P_{2}$. The points $D$ and $P_{1}$ form one pair of conjugates, and $D$ and $P_{2}$ another; hence $\delta_{6}^{\prime}$ has a double point where it meets $h^{\prime}$, the tangents at which are distinct from each other and from $h^{\prime}$. The point is a triple point on $S_{6}^{\prime}$. The surface $S_{6}^{\prime}$ has a composite double curve consisting of $\delta_{6}^{\prime}$ and of $h^{\prime}$, the latter passing through 
a double point on the former. The genus of the composite $c_{7}$ can be found as follows. From an arbitrary point can be drawn 8 lines meeting $\delta_{6}^{\prime}$ twice, accounting for 7 apparent double points, and the line to the double point. The plane determined by the point and $h^{\prime}$ meets $\delta_{6}^{\prime}$ in 4 other points, hence $h=8+4=12, p=3$.

The web $\left|S_{5}\right|$ can be transformed birationally into a web of monoids. Let $\gamma_{3}$ be defined by

$$
\frac{x_{1}}{x_{2}}=\frac{x_{2}}{x_{3}}=\frac{x_{3}}{x_{4}}
$$

Put $Q_{1}=x_{1} x_{3}-x_{2}{ }^{2}, Q_{2}=x_{2} x_{4}-x_{3}{ }^{2}, Q_{3}=x_{1} x_{4}-x_{2} x_{3}$. Consider the transformation defined by

$$
y_{i}=Q_{i} x_{4},(i=1,2,3), y_{4}=\sum u_{i} Q_{i},
$$

where $u_{i}$ is linear in $x_{1}, x_{2}, x_{3}$. The equations define a $(3,3)$ non-involutorial transformation between $(x)$ and $(y)$ in which the fundamental elements in $(x)$ are $\gamma_{3}$ and a plane cubic $C_{3}: x_{4}=0, \sum u_{i} Q_{i}=0$ meeting $\gamma_{3}$ in three points. The image in $(y)$ of the plane $x_{4}=0$ is the point $(0,0,0,1) \equiv O$. Every point of $\gamma_{3}$ is transformed into a straight line, the locus of which is a ruled surface $R_{5}$ of order 5 , trisecants of an elliptic $C_{6}: O^{3}$. The image of a point of $C_{3}$ is a line through $O$, the locus of which is the cubic cone $\Gamma_{3}: C_{6}$.

The image of an arbitrary line of $(x)$ is a cubic curve through $O$; a secant of $\gamma_{3}$ has for image a conic through $O$ and a generator of $R_{5}$; a bisecant of $\gamma_{3}$ goes into a line through $O$ and two generators of $R_{5}$. Thus the congruence of bisecants of $\gamma_{3}$ is transformed into the bundle $O$. Since every line of the congruence meets $S_{5}$ in one point not on $\gamma_{3}$, the image must be a monoid in $(y)$. The complete image of $S_{5}$ is of order 15, but $R_{5}^{2}$ is a component; $\alpha^{2}, \bar{\alpha}^{2}$ go into double basis lines through $O$.

The curve $\beta_{5}$ has for image a composite curve of order 15, consisting of a proper curve $\beta_{7}$ of order 7 and of eight lines on $R_{5}$. Since $\beta_{5}$ meets $x_{4}=0$ in 5 points, $\beta_{7}$ has $O$ for five fold point. The residual $\bar{C}_{6}$ is simple, 


$$
s_{5}: O^{4} \alpha \bar{\alpha} \beta_{7} \bar{C}_{6} .
$$

The variable curve of intersection is a $C_{10}$ having $O^{6}$.

Since the variable $C_{6}$ meets each $\alpha$ in two points, the image $C_{10}$ meets each image $\bar{\alpha}$ in two points, hence the curve lies on a quartic cone of genus 1 , with two fixed double generators, $\left[\beta_{7}, \bar{C}_{6}\right]=6$.

15. Resumption of the Case $p=3$. This completes the enumeration of webs of rational surfaces with plane sections of genus 3, belonging to the first species. (Castelnuovo (9).) The normal surfaces are of order $16-\kappa$, lying in space of $14-\kappa$ dimensions $(\kappa<12)$. Those that are not normal can be projected into one or another of the types just considered. (Clebsch (19), Cremona (24), Sturm (108).)

The $F_{6}$ with a double curve of order 7 , genus 3 , has an actual triple point on both curve and surface. (Bordiga (7), Veronese (110).)

The system $\left|C_{4}\right|: 10 P^{1}$ defines a sextic surface having a double curve $C_{7}, p=3$ with a triple point. The residual curve of order 8 is rational, hence condition (a) is not satisfied. Similarly for surfaces defined by $\left|C_{4}\right|$ having fewer than 10 basis points.

The second species is composed of those surfaces with plane sections of genus 3 which also contain an $\infty^{2}$ system of curves of genus 1 ; this system can be reduced to quartics. (Castelnuovo (9).) If the system of elliptic quartics is composed of plane curves, their planes pass through a point, and the surfaces are quartics having the given point for tacnode.

In every case the surface contains an $\infty^{2} I_{2}$ of points, the lines joining pairs of conjugates being concurrent. From this point the surface can always be projected into a surface of Veronese in $S_{5}$, counted twice. The surface is always rational except when the arithmetic genus is -1 and when the curve of invariant points of the $g_{2}{ }^{1}$ from $O$ consists of four concurrent conics. These cases do not concern us. If the curves of genus 1 lying on the surface are space quartics, 
the surface can be reduced to a quintic of $S_{3}$ having three concurrent double lines, and hence a triple point. The net of quartics is cut from the surface by the net of quadric cones passing simply through the double lines.

The $F_{8}$ of $S_{6}$, represented by $\left|C_{6}\right|: 7 P^{2}$, can be projected into an $F_{4}$ of $S_{3}$ by choosing the four centers of projection on the same elliptic curve lying on it. The result is a quartic of $S_{3}$ with a tacnode and four simple concurrent lines in the tangent plane at the tacnode. (Noether (80), Cremona (24).)

The system of curves $\left|C_{6}\right|: 7 P^{2} 3 P^{1}$ defines an $F_{5}: 3 C_{1}^{2} 3 C_{1}{ }^{1}$, the three double lines being concurrent, and the three simple lines lying in the planes containing a pair of double lines. In the plane representation, let the double points be $A, B, C, D, E, F, G$ and the simple ones $P, Q, R$.

The plane images of the three double lines are $C_{3}: A \ldots$ $G P Q ; C_{3}: A \cdots G P R ; C_{3}: A \cdots G Q R$. From the complete intersection of two surfaces of the system $\left(F_{5}\right)$ the three double lines should be subtracted, hence the plane sections are represented by $\left|C_{12}\right|: A^{4} \cdots G^{4} P Q R$. Thus this characteristic is of grade 29 , genus 13 , dimension 17 . Hence there are nineteen linearly independent surfaces of the system.

In order to secure a subsystem satisfying conditions (a), (b), (c), let $\left(F_{5}\right)$ have an additional basis curve $C_{m}$, of order $m$, genus $p$, meeting the totality of the double lines in $S$ points; then

$$
\begin{aligned}
11 m-2 p+2-5 s & =26, \\
5 m-p+1-2 s & =14,
\end{aligned}
$$

so that $s=m+2, p=3 m-17$. (Sharpe and Snyder (100), p. 71.) The residual intersection of genus 1 is met by any third surface in three points, hence it can be reduced to a cubic through six basis points. It has one of the four forms
$(\alpha)$
$C_{3}: \quad A B C D E F$,
$C_{3}: \quad A B C D E P$,
$C_{3}: \quad A B C D P Q$,

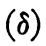
$C_{3}: \quad A B C P Q R$. 
In $(\alpha)$, the residual basis curve $C_{m}$ is

$$
C_{9}: \quad A^{3} \cdots F^{3} G^{4} P Q R .
$$

The corresponding basis curve on the surfaces $|f(y)|$ is $C_{7}, p=4$, meeting each double line in 3 points. Through $O$ can be drawn two other bisecants of $C_{7}$; these lines are fundamental, having five fixed points of intersection with each $f(y)$ of the system. Hence $L_{4}(x)$ has additional double points, and consequently $V$ has a double point.

$(\beta)$. The basis curve is now of order 6 , genus 1 , meeting two of the double lines each in 3 points and the third in 2 points. Hence as before, two additional bisecants can be drawn from $O$; these lines are fundamental, giving rise to double points on $L_{4}(x)$, and hence on $V$.

$(\gamma)$. The basis curve is now composite, consisting of two conics which do not intersect, each meeting all the double lines once, and of a line skew to both conics, meeting one double line. The additional secant of both conics from $O$ accounts for double points on $V$.

$(\delta)$. This case is impossible, as the residual basis curve is of order 4 and genus -5 .

There is one more possiblecase of systems $|f(y)|$ where plane sections of genus 3 exists, namely, that having a plane representation of the form $\left|C_{7}\right| ; 7 P^{2} 2 P_{1}$. (Caporali (8).)

The double basis curve of the system $|f(y)|$ consists of a line $C_{1}$ and of a sextic $\gamma_{6}, p=2$, having two double points on the line. One surface of the system $|f(y)|$ must be composite, hence $L_{4}$ and also $V_{3}$ have double points.

The cases of systems of species 3 and of species 4, Castelnuovo (9), reduce to systems of quartics with point singularities more complicated than in that of type 2 , but without basis lines. (Noether (83).)

The first surface is represented by $\left|C_{6}\right|: 7 P^{2} 4 P^{1}$, all 11 basis points lying on a cubic curve $\gamma$. Let the singular point be $(0,0,0,1)$, and the tangent plane be $x_{1}=0$. Two surfaces of the system meet in a curve $C_{16}$ of order 16 , with 8 branches through $O$, all lying in $x_{1}=0$. The image of a plane section 
through $O$ consists of $\gamma$ and of another cubic with the symbol $C_{3}: A B C D E F G$. The plane representation of $C_{16}$ is $C_{12}: 7 P^{4}$. When this is composite, one component being of genus 1 and variable, it can be reduced to the $C_{3}: A \cdots F$, and the fixed basis curve has for image $C_{9}: A^{3} \cdots F^{3} G^{4}, p=4$. In space the variable curve is a $C_{6}$ with three branches through $O$; the basis curve is then $C_{10}$ with five branches through $O$. Since two trisecants of $C_{10}$ can be drawn through $O$, these lines are fundamental, giving rise to double points on $V_{3}{ }^{3}$.

It was assumed that the variable cubic in the plane representation had for basis points $A, B, C, D, E, F$; if instead one or more be chosen from the simple basis points of the system, practically the same argument may be repeated, with the same conclusion.

Another case is that of $F_{4}$ represented by $\left|C_{7}\right|: P^{39} 9 P^{2}$, all the basis points lying on a cubic curve, and another is the $F_{4}$ represented by $\left|C_{8}\right|: 8 P^{3} P^{2} P^{1}$, the ten basis points lying on a cubic. Both of these can be considered as the preceding case, with the same result.

This completes the case $p=3$, non-hyperelliptic. When the net of curves on each surface of the system is composite, the plane sections of genus 3 are hyperelliptic. In this case the $F_{16}$ is represented by $\left|C_{6}\right|: P^{4} P^{2}$ or by $\left|C_{8-k}\right|: P^{6-k}(3-k)$ $P^{2},(k=0,1,2,3)$, the double points being adjacent to the $P^{6-k}$. They will be considered in connection with the general hyperelliptic case. (De Franchis (25).)

16. General Hyperelliptic Sections. V. From Castelnuovo (10) and Enriques (31) we know that every surface with hyperelliptic plane sections or by sections $S_{n-1}$, if it lies in $S_{n}$, is rational. If the genus of the sections exceeds 1 , the surface contains a rational $\infty^{1}$ system of conics, such that through any point of the surface passes just one conic of the system. If the section is of order $n$ and genus $\pi$, the surface cannot belong to a space larger than $S_{n-\pi+1}$; if it belongs to a space of lower dimensionality, it is the projection of a normal surface of $S_{n-\pi+1}$. 
The planes of the $\infty^{1}$ conics on the surface form a variety of order not larger than $n-\pi-1$.

The surface can be mapped on a plane such that the plane sections (or those of $S_{r-1}$ if in $S_{r}$ ) are represented by curves of order $k$ having a common point of multiplicity $k-2$, and possibly other basis points. Hence if the genus is $\pi$, the maximum order is $4 \pi+4$. Every such surface of lower order can be obtained from a surface of order $4 \pi+4$ of $S_{3 \pi+5}$ from $4 \pi+4-n$ points upon it, and possibly from other points not on the surface.

In order to obtain a subsystem satisfying conditions (a), (b), (c), it is necessary to impose simple basis points in every case. When double points exist among the additional basis points, the curves can be reduced to those of lower order. Several cases of $\left|C_{k}\right|: P^{k-2}$ have been considered in connection with other problems. Let $x$ be the number of simple basis points, $N$ the order of the surface, $m$ the order of its double curve. Then for $(k, x, N, m)(4,8,4,1)$ see $(100)$, p. $61 ;(4,7,5,3)$ see $(100)$, p. $72 ;(4,6,6,8)$ see $(8)$, p. 202 ; $(5,11,5,3)$ see $(100)$, p. $71 ;(5,10,6,7)$ see $(8)$, p. 207.

The system $\left|F_{N}\right|: C_{1}^{N-2} \gamma$ can be treated as in (100) p. 62 , and the monoidal cases of any order necessarily have fundamental lines.

If the assumption made in $\S 8$ is justifiable it follows that the general cubic variety of $S_{4}$ is irrational.

17. Reduction to Monoidal Types. From the preceding representation, follows at once the proof of the following theorem.

All $(1,2)$ quaternary correspondences of genus 1 , in which the quartic surface of branch points has a singular plane and at least one double point not in this plane, can be expressed in terms of $a(1,1)$ correspondence between the points of an $S_{3}{ }^{\prime}$ and the pairs of points of a perspective monoidal involution of order two in another space $S_{3}$.

Let there be a second involution $I_{2}$ in $(x)$, having the same surface of branch points $L_{4}{ }^{\prime}$ in $\left(x^{\prime}\right)$. Since the points of $V_{8}$ 
and of $(x)$ are in $(1,1)$ correspondence, we have in any case $y_{i}=f_{i}\left(x_{1}, x_{2}, x_{3}, x_{4}\right), i=1,2,3,4$. For example, by including the vertex $P$ on $V_{3}$ from which the variety is projected on the double $S_{3}$, we may write

$$
y_{i}=F_{2}(x) x_{i} ; \quad y_{5}=F_{3}(x) .
$$

Let the pairs of points of $I_{2}$ also be represented on $V_{3}$. Since the two surfaces of coincident points $K_{1}$ of $I_{1}$ and $K_{2}$ of $I_{2}$ are each in $(1,1)$ correspondence with $L_{4}{ }^{\prime}$, they can be superposed one on the other.

Then $I_{2}$ is transformed into a second monoidal involution, having a second double point $Q_{x}$ on $K_{2}$ for vertex. The image on $V_{3}$ of $Q_{x}$ is a second double point, hence $L_{4}^{\prime}$ has a second double point. The line joining the double points on $V_{3}$ lies entirely upon it; every plane through it meets $V_{3}$ in a residual conic, image of a line of the bundle $Q_{x}$ in $(x)$. This line contains an infinite number of pairs of conjugate points with double points on $K_{x}$. Hence the conic on $V_{3}$ contains an infinite number of pairs of points forming an involution in which the double points are the residual points of intersection of the plane of the conic with the curve of contact $K_{V}$ of the tangent cone from $P$. Thus, by $I_{2} \mathrm{v}$ every point of $K_{V}$ remains invariant; by projection upon $x_{5}{ }^{\prime}=0$, the associated point on $L_{4}{ }^{\prime}$ is a branch point as before.

If in $(x)$ there exist several involutions having the same quartic surface of branch points with a singular plane, by the above process they can all be reduced to perspective monoidal involutions by the same transformation.

When $L_{4}^{\prime}$ has only one double point not in the singular plane, the web of invariant cubic surfaces in $(x)$ has a simple point and a general $C_{6}, p=4$, for basis elements. It is described as case $D$ of cubics in Sharpe and Snyder (100), p. 58. It does not appear again in a transformed form in the enumeration there given.

If $L_{4}{ }^{\prime}$ has two double points not in the singular plane, $K(x)$ has a second double point, the quadric $F_{2}$ containing $C_{6}$ and 
the cubics of the web touch each other, $C_{6}$ has an actual double point and is now of genus 3 .

Similarly, $L_{4}{ }^{\prime}$ may have 3 or $4, \ldots$ or 10 double points not in its singular plane. In the latter case it is the 16-nodal Kummer surface, and $K$ may be reduced to the symmetroid.

\section{REFERENCES}

(1) Alexander, J. W., On the factorization of Cremona plane transformations, Transactions of this Society, vol. 17 (1915), pp. 295-300.

(2) Aprile, G., Sopra la involuzione non razionale di Enriques, Rassegna di Matematica e Fisica, vol. 1 (1921), pp. 133-136.

(3) Bagnera, G., and De Franchis, M., Sopra le superficie che hanno le coordinate del punto generico esprimibile con funzioni meromorfe quadruplamente periodiche, Rendiconti, Accademia dei Lincei, (5), vol. 161 (1907), pp. 492-498 and pp. 596-603.

(4) - Le superficie algebriche le quali ammettono una rappresentazione parametrica mediante funzioni iperellittiche di due argomenti, Memorie della Società Italiana delle Scienze, (3), vol. 15 (1908), pp. 251343.

(5) Le nombre o de M. Picard pour les surfaces hyperelliptiques et pour les surfaces irregulières de genre zero, Rendiconti di Palermo, vol. 30 (1910), pp. 185-238.

(6) Bertini, E., Ricerche sulle trasformazioni univoche involutorie nel piano, Annali di Matematica, (2), vol. 8 (1877), pp. 11-23 and 146 and 244-286.

(7) Bordiga, G., La superficie del $6^{\circ}$ ordine con 10 rette, nello spazio $R_{\mathbb{4}}$ e le sue projezioni nello spazio ordinario, Memorie, Accademia dei Lincei, (4), vol. 3 (1887), pp. 182-203.

(8) Caporali, E., Sopra $i$ sistemi lineari triplamente infiniti di curve algebriche piane, Collectanea Mathematica in Memoriam Dominici Chelini, Mediolani, 1881; Memorie di Geometria, Napoli, 1888.

(9) Castelnuovo, G., Sulle superficie algebriche le cui sezioni sono curve di genere 3, Atti, Accademia di Torino, vol. 25 (1889-1890), pp. 695-715.

(10) Sulle superficie le cui sezioni piani sono curve iperellittiche, Rendiconti di Palermo, vol. 4 (1890), pp. 73-88. , Sulla razionalitd delle involuzioni piane, Rendiconti, Accademia dei Lincei, (5), vol. 22 (1893), pp. 205-209; Mathematische Annalen, vol. 44 (1893), pp. 125-155.

(12) - Sulle superficie algebriche le cui sezioni piane sono curve ellittiche, Rendiconti, Accademia dei Lincei, (5), vol. $3_{1}$ (1894), pp. 59-61. 
(13) - Sulle superficie di genere zero, Memorie della Società Italiana delle Scienze, (3), vol. 10 (1896), pp. 103-123.

(14) , Le trasformazioni geometriche del gruppo cremoniano nel piano, Atti, Accademia di Torino, vol. 36 (1901), pp. 861-874.

(15) Castelnuovo, G., and Enriques, F., Sulle condizioni di razionalitd di piani doppi, Rendiconti di Palermo, vol. 14 (1900), pp. 290-302.

(16) - Sopra alcune questioni fondamentali nella teoria delle superficie algebriche, Annali di Matematica, (3), vol. 6 (1901), pp. 165-225.

(17) Chisini, O., Sul teorema di Noether, Memorie, Accademia in Modena, (5), vol. 6 (1921-1922), pp. 7-13.

(18) Clebsch, A., Ueber die Anwendung der Abel'schen Funktionen in der Geometrie, Journal für Mathematik, vol. 64 (1864), pp. 189-243.

(19) Zur Theorie der Cremonaschen Transformationen, Mathematische Annalen, vol. 4 (1871), pp. 490-496.

(20) Coble, A. B., Point sets and allied Cremona groups, Transactions of this Society, vol. 17 (1914), pp. 345-385.

(21) - The ten nodes of the rational sextic and of the Cayley symmetroid, American Journal of Mathematics, vol. 41 (1919), pp. 243-265. Cremona transformations and applications to algebra, geometry, and modular functions, this Bulletin, vol. 28 (1922), pp. 329-364.

(23) Comessatti, A., Sulle superficie razionali reali, Rendiconti, Accademia dei Lincei, (5), vol. 20 2 (1911), pp. 597-602; Mathematische Annalen, vol. 73 (1912), pp. 1-72.

(24) Cremona, L., Ueber die Abbildung algebraischer Flächen, Göttinger Nachrichten, 1871, pp. 129-143; reprinted in extended form Mathematische Annalen, vol. 4 (1871), pp. 213-230.

(25) De Franchis, M., Riduzione dei sistemi lineari $\infty^{k}$ di curve piane di genere $k$ per $k>1$, Rendiconti di Palermo, vol. 13 (1899), pp. 130-160.

(26) Del Pezzo, P., Sulle superficie del $N^{m o}$ ordine immerse nello spazio di $N$ dimensioni, Rendiconti di Palermo, vol. 1 (1884-1885), pp. 24-71.

(27) De Paolis, R., Le trasformazioni doppie dello spazio, Memorie, Accademia dei Lincei, (4), vol. 1 (1885), pp. 576-608.

(28) Enriques, F., Sugli spazi pluritangenti delle varietà cubiche generali appartenenti allo spazio a quattro dimensioni, Giornale di Matematiche, vol. 31 (1893), pp. 31-35.

(29) - Sui gruppi continui di trasformazioni cremoniane nel piano, Rendiconti, Accademia dei Lincei, (5), vol. $2_{1}$ (1893), pp. 468-473.

(30) - Sui sistemi lineari di superficie algebriche le cui intersezioni variabili sono curve ellittiche, Rendiconti, Accademia dei Lincei, (5), vol. $3_{1}$ (1894), pp. 481-487, and pp. 536-543.

(31) , Sui sistemi lineari di superficie algebriche ad intersezioni iperellittiche, Mathematische Annalen, vol. 46 (1895), pp. 179-199.

(32) - Sui piani doppi di genere zero, Memorie della Società Italiana delle Scienze, (3), vol. 10 (1896), pp. 201-221. 
(33) Sulle irrazionalità da cui può farsi dipendere la risoluzione d'un'equazione algebrica $f(x y z)=0$ con funzioni razionali di due parametri, Mathematische Annalen, vol. 49 (1897), pp. 1-23.

(34) - Intorno ai fondamenti della geometria sopra le superficie algebriche, Atti, Accademia di Torino, vol. 37 (1901), pp. 19-40.

(35) - Sulle superfici di genere geometrico zero, Rendiconti di Palermo, vol. 20 (1905), pp. 1-33.

(36) - Sopra un'involuzione non razionale dello spazio, Rendiconti, Accademia dei Lincei, (5), vol. 21 (1912), pp. 81-83.

(37) - Intorno alla risoluzione razionale di una classe di equazioni algebriche fra quattro variabili, Annali di Matematica, (3), vol. 20 (1913), pp. 109-111.

(38) Enriques, F., and Fano, G., I gruppi continui di trasformazioni cremoniane dello spazio, Annali di Matematica, (2), vol. 26 (1897), pp. 59-98.

(39) Enriques, F., and Severi, F., Mémoire sur les surfaces hyperelliptiques, Acta Mathematica, vol. 32 (1909), pp. 283-392; vol. 33 (1909), pp. 321-403.

(40) Fano, G., Sulle superficie algebriche con infinite trasformazioni proiettive in sè stesse, Rendiconti, Accademia dei Lincei, (5), vol. 41 (1895), pp. 149-156.

(41) - Sulle equazioni differenziali lineari di ordine qualunque, che definiscono curve contenute in superficie algebriche, Rendiconti, Accademia dei Lincei, (5), vol. 4 (1895), pp. 322-330.

(42) - Sulle varietà algebriche con un gruppo continuo non integrabile di trasformazioni proiettive in sè, Memorie, Accademia di Torino, (2), vol. 46 (1895-1896), pp. 187-218.

(43) - Sulle varietà algebriche dello spazio a quattro dimensioni con un gruppo continuo integrabile di trasformazioni proiettive in sè, Atti, Istituto Veneto, (7), vol. 7 (1895-1896), pp. 1069-1108.

(44) - Sulle superficie algebriche con un gruppo continuo transitivo di trasformazioni proiettive in sè, Rendiconti di Palermo, vol. 10 (1896), pp. 1-15.

(45) , Sui gruppi continui di trasformazioni cremoniane del piano e sopra certi gruppi di trasformazioni proiettive, Rendiconti di Palermo, vol. 10 (1896), pp. 16-29.

(46) - I gruppi continui primitivi di trasformazioni cremoniane dello spazio, Atti, Accademia di Torino, vol. 33 (1898), pp. 480-504.

(47) - I gruppi di Jonquières generalizzati, Memorie, Accademia di Torino, (2), vol. 48 (1898), pp. 221-278.

(48) - Sopra alcuni gruppi continui imprimitivi di trasformazioni puntuali dello spazio, Rendiconti, Accademia dei Lincei, (5), vol. $7_{1}$ (1898), pp. 302-308. 
(49) , Le trasformazioni infinitesime dei gruppi cremoniani tipici dello spazio, Rendiconti, Accademia dei Lincei, (5), vol. $7_{1}$ (1898), pp. 332-340.

(50) , Sulle equazioni differenziali lineari che appartengono alla stessa specie delle loro aggiunte, Atti, Accademia di Torino, vol. 34 (18981899), pp. 888-911.

(51) -, Sulle equazioni differenziali lineari del $5^{\circ}$ e del $6^{\circ}$ ordine, le cui curve integrali sono contenute in una quadrica, Atti, Accademia di Torino, vol. 34 (1898-1899), pp. 415-445.

(52) - Osservazioni sopra alcuni equazioni differenziali lineari, Rendiconti, Accademia dei Lincei, (5), vol. 81 (1899),pp.285-291.

(53) - Un teorema sulle varietd algebriche a tre dimensioni con infinite trasformazioni proiettive in sè, Rendiconti, Accademia dei Lincei, (5), vol. 81 (1899), pp. 562-565.

(54) - Sulle equazioni differenziali lineari del $5^{\circ}$ ordine le cui curve integrali sono contenute in una varietd algebrica, Rendiconti, Istituto Lombardo, (2), vol. 32 (1899), pp. 843-866.

(55) - Ueber lineare homogene Differentialgleichungen mit algebraischen Relationen zwischen den Fundamentallösungen, Mathematische Annalen, vol. 53 (1900), pp. 493-590.

(56) - Sulle superficie algebriche contenute in una varietd cubica dello spazio a quattro dimensioni, Atti, Accademia di Torino, vol. 39 (1904), pp. 597-615.

(57) - Sul sistema $\infty^{2}$ di rette contenute in una varietd cubica generale dello spazio a quattro dimensioni, Atti, Accademia di Torino, vol. 39 (1904), pp. 778-792.

(58) - Ricerche sulla varietà cubica generale dello spazio a quattro dimensioni e sopra i suoi spazi pluritangenti, Annali di Matematica, (3), vol. 10 (1904), pp. 251-258.

(59) - Sopra alcune varietd algebriche a tre dimensioni aventi tutti $i$ generi nulli, Atti, Accademia di Torino, vol. 43 (1908), pp. 973-984.

(60) - Sulle varietd algebriche a tre dimensioni a superficie sezioni razionali, Annali di Matematica, (3), vol. 24 (1915), pp. 49-88.

(61) - Osservazioni sopra alcune varietà non razionali aventi tutti i generi nulli, Atti, Accademia di Torino, vol. 50 (1914-1915), pp. 10671072.

(62) _- Sulle varietd algebriche a tre dimensioni aventi tutti $i$ generi nulli, Bologna Congress of Mathematicians, 1928.

(63) Guccia, G. B., Sulle superficie algebriche le cui sezioni piane sono unicursali, Bulletin de la Société Mathématique, vol. 14 (1885), p. 137; Rendiconti di Palermo, vol. 1 (1884-1885), pp. 165-168.

(64) Hollcroft, T. R., On plane involutions of order 4, American Journal of Mathematics, vol. 44 (1922), pp. 163-171.

(65) Howe, A. M., On the classification of plane $(1,3)$ transformations, American Journal of Mathematics, vol. 41 (1919), pp. 25-48. 
(66) Hudson, H. P., On the composition of Cremona space transformations, Rendiconti di Palermo, vol. 35 (1913), pp. 286-288.

(67) - Cremona Transformations in Plane and Space, Cambridge, University Press, 1927.

(68) Hudson, R. W. H. T., Kummer's Quartic Surface, Cambridge, University Press, 1905.

(69) Kantor, S., Sur une théorie des courbes et des surfaces admettant des correspondances univoques, Comptes Rendus, vol. 100 (1885), pp. 343-345.

(70) - Premiers fondaments pour une théorie des transformations périodiques univoques, Atti, Accademia di Napoli, (2), vol. 1 (1883), pp. 1-264; extensive abstract in Journal für Mathematik, vol. 114 (1895), pp. 50-108.

(71) Lüroth, F., Beweis eines Satzes über rationale Kurven, Mathematische Annalen, vol. 9 (1875), pp. 163-165.

(72) Marletta, G., Di una classe di forme dell'S 4 ognuna rappresentabile nelle coppie di un'involuzione dell' $S_{3}$, Rendiconti, Accademia dei Lincei, (5), vol. 271 (1918), pp. 371-374.

(73) Mlodzieioski, B., On the composition of plane Cremona transformations, (in Russian), Recueil, Moscow Mathematical Society, vol. 29 (1914), pp. 269-275.

(74) - Sur la théorie des transformations Crémoniennes, Recueil, Moscow Mathematical Society, vol. 31 (1916), pp. 7-34.

(75) - Tables des nombres Crémoniens des 21 premiers ordres, Recueil, Moscow Mathematical Society, vol. 31, pp. 35-77.

(76) - Sur les systemes linéaires de courbes liêes aux solutions arithmétiques des équations Crêmoniennes, Recueil, Moscow Mathematical Society, vol. 31 (1924), pp. 341-344.

(77) Montesano, D., Una estensione del problema della proiettivita a gruppidi complessie di congruenze lineari di rette, Annali di Matematica, (3), vol. 1 (1898), pp. 313-358.

(78) - Su la teoria generale delle corrispondenze birazionali fra $i$ punti dello spazio, Atti, Accademia di Napoli, (2), vol. 17 (1926), pp. 1-25.

(79) - Su alcuni problemi fondamentali nella teoria della corrispondenze cremoniane, Rendiconti, Accademia di Napoli, (3), vol. 34 (1928), pp. 42-50, and pp. 89-97, and pp. 123-136.

(80) Noether, M., Ueber Flächen, welche Schaaren rationaler Curven besitzen, Mathematische Annalen, vol. 3 (1870), pp. 161-227; vol. 5 (1872), pp. 635-639.

(81) —_ Ueber die ein-zweideutigen Ebenentransformationen, Erlangen physico-medicinische Societäts, Sitzungsbericht, vol. 10 (1878), pp. 81-86.

(82) Zur Theorie des eindeutigen Entsprechens algebraischer Gebilde, Mathematische Annalen, vol. 8 (1874-1875), pp. 495-533. 
(83) - Ueber die rationalen Flächen vierter Ordnung, Mathematische Annalen, vol. 33 (1888), pp. 546-571.

(84) Pannelli, M., Sopra gli invarianti di una varieta algebrica a tre dimensioni rispetto alle trasformazioni birazionali, Rendiconti, Accademia dei Lincei, (5), vol. 15 (1906), pp. 483-489.

(85) Picard, E., Sur une classe des surfaces algébriques, Bulletin de la Société Philomathique de France, (7), vol.2 (1878), pp. 127-131; amplified in Journal für Mathematik, vol. 100 (1886), pp. 71-78.

(86) Scorza, G., Le varietd a curve sezioni ellittiche, Annali di Matematica, (3), vol. 15 (1908), pp. 217-273.

(87) - Le superficie a curve sezioni di genere tre, Annali di Matematica, (3), vol. 16 (1909-1910), pp. 255-326.

(88) Segre, C., Sui sistemi lineari di curve piane algebriche di genere $p$, Rendiconti di Palermo, vol. 1 (1884-1885), pp. 217-221.

(89) - Sulle varieta cubiche dello spazio e quattro dimensioni, Memorie, Accademia di Torino, (2), vol. 39 (1889), pp. 3-48.

(90) — Un'osservazione relativa alla reducibilità delle trasformazioni Cremoniane e dei sistemi lineari di curve piane per mezzo di trasformazioni quadratiche, Atti, Accademia di Torino, vol. 36 (1901), pp. 645-651.

(91) Severi, F., Rappresentazione di una forma qualunque per combinazione lineare di piì altre, Rendiconti, Accademia dei Lincei, (5), vol. 11 (1902), pp. 105-113.

(92) Sulle intersezioni di varietà algebriche e sopra i loro caratteri e singolarita proiettive, Memorie, Accademia di Torino, (2), vol. 52 (1903), pp. 61-118.

(93) Su alcune questioni di postulazione, Rendiconti di Palermo, vol. 17 (1903), pp. 73-103.

(94) - Osservazioni varie di geometria sopra una superficie e sopra una varietd, Atti, Istituto Veneto, vol. 65 (1905-1906), pp. 625-643.

(95) - Una proprieta delle forme algebriche prive di punti multipli, Rendiconti, Accademia dei Lincei, (5), vol. 152 (1906), pp. 691696.

(96) - Intorno alle superficie iperellittiche, Rendiconti, Accademia dei Lincei, (5), vol. 161 (1907), pp. 443-453.

(97) - Alcune proprietd fondamentali per la geometria sulle varietd algebriche, Rendiconti, Accademia dei Lincei, (5), vol. 162 (1907), pp. 337-344. Intorno alle superficie iperellittiche, irregolari, Rendiconti, Accademia dei Lincei, (5), vol. 17 (1908), pp. 4-9.

(99) Sharpe, F. R., The problem of plane involutions of order $t>2$, American Journal of Mathematics, vol. 50 (1928), pp. 627-635.

(100) Sharpe, F. R., and Snyder, V., Certain types of involutorial space transformations, Transactions of this Society, vol. 20 (1919), pp. 185-202; vol. 21 (1920), pp. 52-78. 
(101) Snyder, V., An application of the $(1,2)$ quaternary correspondence to the Weddle and Kummer surface, Transactions of this Society, vol. 12 (1910), pp. 354-366.

(102) Birational transformations of the cubic variety in four-dimensional space, Rendiconti di Palermo, vol. 38 (1914), pp. 344-352.

(103) —_ Problems in involutorial transformations in space, this Bulletin, vol. 30 (1923), pp. 101-124.

(104) Un'involuzione appartenente alla varietà cubica dello spazio di quattro dimensioni, Giornale di Matematiche, vol. 62 (1924), pp. 125-128.

(105) - Non monoidal involutions which contain a web of invariant monoids, Annals of Mathematics, (2), vol. 26 (1925), pp. 165-172.

(106) - Selected Topics in Algebraic Geometry, Bulletin No. 63, National Research Council, 1928. By A. B. Coble, Arnold Emch, S. Lefschetz, F. R. Sharpe, C. H. Sisam, and the chairman, V. Snyder.

(107) _- - Involutorial space transformations contained multiply in a linear line complex, Bologna Congress of Mathematicians, 1928.

(108) Sturm, R., Ueber die Flächen mit einer endlichen Anzahl von (einfachen) Geraden, vorzugsweise die der vierten und fïften Ordnung, Mathematische Annalen, vol. 4 (1871), pp. 249-283.

(109) Veneroni, E., Sopra certe congruenze di rette e sopra alcune proprietd dei fasci di un complesso cubico generale, Rendiconti, Istituto Lombardo, (2), vol. 31 (1898), pp. 1072-1088.

(110) Veronese, G., Behandlung der projektivischen Verhältnisse der Räume von verschiedenen Dimensionen durch das Princip des Projecirens und Schneidens, Mathematische Annalen, vol. 19 (1881-1882), pp. 161-224.

(111) Voss, A., Ueber Complexe und Congruenzen, Mathematische Annalen, vol. 9 (1876), pp. 55-144.

(112) Wiman, A., Zur Theorie der endlichen Gruppen von birationalen Transformationen in der Ebene, Mathematische Annalen, vol. 48 (18961897), pp. 195-240.

\section{CoRnell University}

